\title{
MUNICÍPIOS E PROPOSTAS VULNERÁVEIS: UMA ANÁLISE DO EDITAL 2013 DO PROGRAMA ESPORTE E LAZER DA CIDADE (PELC)
}

\author{
Edmilson Santos dos Santos \\ Universidade Federal do Vale do São Francisco, Petrolina, Pernambuco, Brasil. \\ Fernando Augusto Starepravo \\ Universidade Estadual de Maringá, Maringá, Paraná, Brasil. \\ Vilde Gomes de Menezes \\ Universidade Federal de Pernambuco, Recife, Pernambuco, Brasil. \\ Esdras Henrique Rangel de Melo \\ Universidade Federal de Pernambuco, Recife, Pernambuco, Brasil.
}

\begin{abstract}
Resumo
As análises de implementação do Programa Esporte e Lazer da Cidade recusam proponentes que não obtiveram sucesso na apresentação de suas propostas. Assim, o objetivo do estudo foi traçar um perfil das prefeituras que não alcançaram 50 pontos no Edital do PELC/2013. Através da estatística descritiva, o estudo traçou comparativos entre essas prefeituras e quatro marcadores de vulnerabilidade: pertencer ao semiárido brasileiro, possuir baixo Índice de Desenvolvimento Humano, ser município com baixo perfil demográfico e ser dependente do Fundo de Participação dos Municípios. A falta de capacidade técnica é um dos marcos das desigualdades entre os municípios brasileiros e aumenta as chances de proponentes vulneráveis não ultrapassarem a nota de corte estabelecida no estudo.
\end{abstract}

Palavras-chave: Políticas Públicas. Esporte. Lazer.

\section{MUNICIPALITIES AND VULNERABLE PROPOSALS: AN ANALYSIS OF THE PUBLIC NOTICE 2013 OF PROGRAM ESPORTE E LAZER DA CIDADE (PELC)}

\begin{abstract}
Abtract: The analyzes of the implementation of the Program Esporte e Lazer da Cidade exempt proponents who were not successful in presenting their proposals. Thus, the objective of the study was to outline a profile of municipalities that did not reach 50 points in the PELC public notice 2013. Through descriptive statistics, the study drew comparisons between these prefectures and four vulnerability markers: they belong to the Brazilian semi-arid region, have a low HDI, be a municipality with a low demographic profile and be dependent on the Municipal Participation Fund. The lack of technical capacity is one of the milestones of the inequalities between Brazilian municipalities and increases the chances of vulnerable proponents not to exceed the cut grade established in the study.
\end{abstract}

Keywords: Public Policy. Sport. Leisure. 


\title{
MUNICIPIOS Y PROPUESTAS VULNERABLES: UN ANÁLISIS DEL EDITAL 2013 DEL PROGRAMA DEPORTE Y OCIO DE LA CIUDAD (PELC)
}

\begin{abstract}
Resumen
Los análisis sobre la implementación del Programa Deporte y Ocio de la Ciudad dispensan a los proponentes que no obtuvieron éxito en la presentación de sus propuestas. Así, el objetivo del estudio fue trazar un perfil de las alcaldías que no alcanzaron 50 puntos en el Edicto del PELC / 2013. A través de la estadística descriptiva el estudio trazó comparativos entre esas prefecturas y cuatro marcadores de vulnerabilidad: pertenecer al semiárido brasileño, poseer bajo IDH, ser municipio con bajo perfil demográfico y ser dependiente del Fondo de Participación de los Municipios. La falta de capacidad técnica es uno de los marcos de las desigualdades entre los municipios brasileños y aumentan las posibilidades de proponentes vulnerables de no sobrepasar la nota de corte establecida en el estudio.
\end{abstract}

Palabras clave: Políticas Públicas. Deporte. Ocio.

\section{Introdução}

Estados federados que centralizam a formulação de políticas sociais criam regras e procedimentos com o objetivo de diminuir desigualdades, a fim de fazer alocações, principalmente de recursos, em direção às regiões e municípios de menor desenvolvimento (ARRETCHE, 2010).

No Brasil, esse compromisso da federação está previsto no Inciso III do Art. 3 da Constituição Federal/1988 (BRASIL, 1988): reduzir as desigualdades sociais e regionais. Nesse sentido, está posto que cabe ao governo federal, no âmbito da federação, através de suas políticas públicas, promover a equidade social ${ }^{1}$.

No campo prático, isso significa aportar recursos onde há maior carência deles, seja de forma pecuniária, diretamente às famílias necessitadas (como o Bolsa Família), na forma de transferências constitucionais (como o Fundo de Participação dos Municípios) ou de serviços [como o Programa Esporte e Lazer da Cidade (PELC)], de forma a combater as desigualdades (TORRES; MARQUES; FERREIRA; BITAR, 2003; ARRETCHE, 2004). Entretanto, essa operação exige certas ferramentas para alocação dos recursos que estão diretamente relacionadas com a capacidade e interesse de coordenação por parte do governo federal.

O Programa Esporte e Lazer da Cidade (PELC) foi formulado pela hoje denominada Secretaria Nacional de Esporte, Educação, Lazer e Inclusão Social (SNELIS) do Ministério do Esporte (ME), em 2003, com o objetivo de proporcionar atividades físicas, culturais e de lazer à população. Como programa esportivo da área social, seu compromisso também deve estar associado à diminuição da desigualdade. O PELC é um programa de descentralização top-down ${ }^{2}$, no qual os parceiros se comprometem em desenvolver ações formuladas pelo governo federal. A decisão do parceiro está entre descentralizar ou não o PELC.

A Secretaria Nacional de Esporte, Educação, Lazer e Inclusão Social (SNELIS) lançou em 2013 o edital para Implantação e Desenvolvimento de Núcleos de Esporte Recreativo e de Lazer (ERL) com o objetivo de implantar três tipos de núcleo: núcleo urbano (NU) e núcleos

$1 \mathrm{O}$ princípio da equidade é basilar à oferta da política pública, principalmente as sociais, (MEDEIROS; DINIZ, 2008), constituindo, como sinaliza Kerstenetzky (2002), espaço para atuação diferenciada a fim de diminuir desigualdades. Portanto, o alvo principal das políticas sociais deve ser a diminuição da desigualdade de acesso a determinados bens, principalmente os escassos.

2 A descentralização top-down acontece quando um ente federado aceita descentralizar uma política desenhada por algum ente federado superior. Difere da descentralização bootom-up, que é quando um ente local descentraliza uma política desenhada formulada e financiada por ele (SABATIER, 1986). 
para povos e comunidades tradicionais (NPCT), na Chamada Pública 001 e núcleo vida saudável (NVS), na Chamada Pública 002.

Para o Edital de 2013, foi vetada a participação de governos estaduais, do Distrito Federal, de entidades sem fins lucrativos e das prefeituras que tiveram seus pareceres de cumprimento do objeto (descentralizações anteriores do PELC) reprovados. Nesse caso, seus principais implementadores seriam os governos municipais (proponentes). Cada proponente poderia apresentar um único projeto.

No Edital de Chamada Pública No 001/2013 está expresso no item 2.2.2 que o programa deve "suprir a carência de políticas públicas e sociais". Ou seja, suprir carências deve estar articulado com o que está previsto no Inciso III do Art. 3 da CF/88. A chamada pública, que explora o elemento concorrencial entre os proponentes, foi a alternativa encontrada pelo Ministério do Esporte para implantação e desenvolvimento de Núcleos de Esporte Recreativo de Lazer nos Municípios do PELC. Os concorrentes disputam entre si para identificar quais as melhores propostas.

Como destaca Oliveira (2006), é na implementação da política pública que se pode perceber com muita nitidez os limites e as fragilidades da política. A competição entre os proponentes foi feita com base em 11 critérios técnicos básicos para seleção das propostas: (1) objetivo geral; (2) objetivos específicos; (3) justificativa do projeto; (4) quadro resumo do pleito; (5) metas do projeto; (6) atividades sistemáticas; (7) atividades assistemáticas; (8) mobilização e inscrição dos participantes; (9) divulgação do projeto; (10) formação continuada; (11) acompanhamento do projeto. O décimo segundo critério era voltado aos municípios que tinham convênios anteriormente pactuados.

A proposta deve ser elaborada por um burocrata e expressar sua capacidade técnica na apresentação. Os projetos recebiam uma pontuação que chegava no máximo a 100 . O peso de cada critério variou de 0,7 a 0,9 . Cada critério foi avaliado conforme os três níveis que valoravam as propostas: baixo, médio e alto. Todos os onze critérios garantem, em tese, paridade entre os municípios.

Os critérios políticos do programa (que priorizam atores vulneráveis) aparecem apenas e tão somente nos critérios de desempate, que são: (a) "ausência de órgão ou conselho municipal de esporte e lazer que exerça e desenvolva as funções relativas" - em ambientes mais frágeis institucionalmente o programa poderia vir a ser um bom estimulador da participação dos atores societais na valorização da temática esportiva; (b) "municípios localizados nas regiões brasileiras do Norte e do Centro-Oeste do país que apresentam número reduzido de parcerias vigentes, ou que não possuem o desenvolvimento da ação"apesar da omissão quanto à região Nordeste, que apresenta inúmeros indicadores de vulnerabilidade, esse critério contempla duas regiões brasileiras de menor desenvolvimento social; (c) "municípios que possuem menos de 50 mil habitantes".

Os municípios menores são aqueles mais dependentes das transferências constitucionais para garantirem patamares mínimos de políticas públicas, destaca-se nesse caso o Fundo de Participação dos Municípios (FPM); (d) "municípios com mais de 50 mil habitantes localizados nas áreas prioritárias no âmbito da PNDR, da SUDENE, SUDAM e SUDECO”. O primeiro é uma política e os outros são órgãos criados pelo governo federal para formularem (ou coordenarem) políticas que visam à diminuição das desigualdades sociais; (e) Índice de Desenvolvimento Humano (IDH). Ou seja, a consideração do IDH se deve ao fato de que diversas políticas, com vistas à diminuição da desigualdade social, tinham esse como critério distribuidor de benefícios.

Os critérios, que vamos chamar de políticos, definem a priori uma vulnerabilidade a ser valorizada e sua definição independe da burocracia governamental. Se não houver empate, são os critérios técnicos que irão balizar as propostas. 
Podemos observar que os critérios de desempate anunciam, em tese, as prioridades políticas das ações coordenadas pelo governo federal. No Inciso 3 do Art. 3 da CF/88 está explícito que as políticas coordenadas por ele devem reduzir as desigualdades sociais e regionais. A redução não se fará pelos critérios técnicos (de competência na elaboração do projeto), mas pelos critérios de desempate.

Vieira et al. (2017) apontam, diante dos resultados da descentralização do PELC, que o IDH poderia melhorar o desempenho do programa ao priorizar os municípios mais vulneráveis. Dada a similaridade das estratégias dos programas PELC e PST, estudo de Santos, Starepravo e Souza Neto (2015) identificou vazios assistenciais na região Nordeste, afetando os municípios que deveriam ser alvos prioritários do programa, mas que estavam fora de sua alça de implementação.

São os critérios apresentados como de viés político que permitem combater a desigualdade social e regional, ao garantir que as transferências negociadas/voluntárias priorizem as unidades subnacionais (municípios) mais vulneráveis (PAES; SIQUEIRA, 2008). Eles (municípios) são fortemente dependentes da capacidade do governo federal de ofertar políticas públicas (PAES; SIQUEIRA, 2008; GOMES; DOWELL, 2000; SOUZA, 2001).

Levando em consideração que a distribuição da capacidade técnica (elaborar projeto) pode ser uma causa da desigualdade entre os municípios (MELO, 2005), sua utilização como critério prioritário pode produzir um efeito contrário ao proposto pela política: excluir municípios vulneráveis.

O desenho do PELC negligencia e descarta a evidência que a capacidade técnica é um dos elementos que produz e reproduz as desigualdades de acesso às políticas públicas, principalmente nos municípios menores do interior do país. Ou seja, a falta de capacidade técnica explica parte da desigualdade de distribuição das políticas públicas no Brasil e não é um critério razoável para se alcançar o que está prevista como função da União para com a federação. Isso pode acarretar efeitos colaterais indesejados (MELO, 1996; TREVISAN; BELLEN, 2008), como o aumento da desigualdade entre as regiões (ALMEIDA, 1995; ARRETCHE; VAZQUES; GOMES, 2012; ARRETCHE, 1999; MELO, 1996).

Nos trabalhos produzidos que fazem avaliação dessa política pública, é marcante a ausência de análise do desempenho dos editais do PELC. Dentre os trabalhos que procuram fazer a avaliação do programa temos: Souza et al (2010), analisando o sistema de avaliação e monitoramento do programa; Vieira (2010), que buscou fazer uma avaliação global do programa; Castro (2016), que analisou o orçamento do programa; e Starepravo e Marchi Júnior (2013), que analisaram o processo de implementação do PELC. Já Areias e Borges (2011) avaliaram o PELC a partir da sua eficiência, eficácia e efetividade social e Santos (2017) fez uma análise do desempenho das propostas no Edital de 2012.

A análise sobre aqueles proponentes que não tiveram suas propostas classificadas para serem implementadas, principalmente para um programa que propõe a inclusão e democratização do esporte e do lazer, torna-se relevante, pois pode ser capaz de apresentar os limites do modelo de descentralização do Programa Esporte e Lazer da Cidade do Edital na Chamada Pública No 001-002/2013.

Dada a importância do programa na diminuição das desigualdades sociais, torna-se importante avaliar aquelas propostas que tiveram pontuações abaixo de 50 pontos, rendimento considerado insuficiente para garantir a implementação do programa. Nenhum projeto foi implementado com menos de 50 pontos. Torna-se importante questionar: qual é o perfil dos municípios que não alcançaram 50 pontos devido à sua vulnerabilidade?

Nesse sentido, o presente estudo, de caráter descritivo-exploratório, teve como objetivo verificar o perfil dos municípios que não alcançaram 50 pontos de nota nas duas chamadas (001 e 002) do Edital de 2013 do PELC a partir de variáveis que apontam 
vulnerabilidades: (a) semiárido, (b) IDH, (c) classificação quanto ao tamanho das populações e (d) dependência do Fundo de Participação dos Municípios (FPM).

\section{Material E Métodos}

O estudo, de caráter descritivo-exploratório, teve como objetivo principal traçar perfil dos municípios que não alcançaram 50 pontos nas duas chamadas (001 e 002) do Edital de 2013 do PELC a partir de quatro marcadores de vulnerabilidade. Como objetivo específico, buscamos identificar a grau de não adesão ao programa por marcador de vulnerabilidade.

Estabelecemos a nota de corte de 50 pontos por dois motivos: é a metade do total que pode ser alcançado, considerada como pontuação insuficiente por qualquer critério que possamos estabelecer e nenhuma proposta foi implementada com nota inferior a 50 pontos. Portanto, são propostas que não concorrem pela implementação do programa.

Houve duas avaliações das propostas. Uma buscando identificar a nota do proponente e sua inclusão ou não em critérios de vulnerabilidade. Outra, a taxa de não adesão de cada vulnerabilidade. Esse último permite identificar o percentual dos municípios que deveriam ser proponentes privilegiados (pois possuem alguma vulnerabilidade) e que não apresentaram propostas ao edital.

Os quatro marcadores de vulnerabilidades escolhidos foram: pertencer ao semiárido brasileiro, possuir baixo IDH, ser município com baixo perfil demográfico e ser dependente do FPM. Essas variáveis foram escolhidas porque elas apresentam marcadores claros de vulnerabilidade reconhecida dos municípios e permitem análise comparativa nacional (possuem dados nacionais).

Para a sua consecução, estabelecemos o seguinte roteiro:

1) Identificar todos os proponentes e suas respectivas notas publicizadas pelo Ministério do Esporte em seu site. Resultado final - Classificados PELC Núcleos Urbanos ${ }^{3}$. Das cinco cidades que apresentaram propostas para dois núcleos diferentes, extraímos a proposta de menor valor $(\mathrm{n}=992)$.

2) Elaborar o banco de dados dos 5.570 municípios brasileiros com informações sobre as variáveis aqui analisadas, com dados extraídos do site do Instituto Brasileiro de Geografia e Estatística (IBGE).

3) Os níveis de vulnerabilidade foram estabelecidos da seguinte forma:

(a) Quanto à delimitação do semiárido, os dados foram baseados no documento "Nova Delimitação do Semiárido Brasileiro", produzido pelo Ministério da Integração Nacional disponível no site do ministério ${ }^{4}$;

(b) Para o IDH, considerou-se vulneráveis os municípios que se encontram com o nível Muito Baixo (de 0,00 a 0,499) e Baixo (de 0,500 a 0,599);

(c) Considerou-se para a classificação do tamanho da população aqueles municípios com até 20.000 habitantes no Censo de 2010 população; (d) o percentual de dependência do Fundo de Participação dos Municípios (FPM) foi estabelecido pelo percentual do FPM da

\footnotetext{
3Disponíveis em:

<http://www.esporte.gov.br/arquivos/snelis/RESULTADO\%20FINAL\%20CLASSIFICADOS\%20PELC\%20N CLEOS\%20URBANOS.pdf $>$ ); Resultado final - Classificados PELC Povos de Comunidades Tradicionais (<http://www.esporte.gov.br/arquivos/ snelis/PELC2013/RESULTADO\%20FINAL\%20CLASSIFICADOS\%20PELC\%20POVOS\%20DE\%20COMUNI DADES\%20TRADICIONAIS.pdf >) e Resultado final - Classificados Vida Saudável

(<http://www.esporte.gov.br/arquivos/snelis/RESULTADO $\% 20$ FINAL\%20CLASSIFICADOS\%20VIDA\%20SAUDVEL.pdf $>$ )

4 Disponíveis em: <http://www.mi.gov.br/c/document_library/get_file?uuid=0aa2b9b5-aa4d-4b55a6e182faf0762763\&groupId $=24915>$.
} 
soma da arrecadação municipal (IPTU, ISS e ITBI ${ }^{5}$ ) e transferência constitucional do FPM $^{6}$. Aqueles municípios cuja arrecadação própria fica entre $0 \%$ e $20 \%$ do FPM foram considerados por nós como dependentes do FPM. As três últimas informações foram coletadas diretamente no site do IBGE.

O Edital de 2013 apresentou três modalidades de participação: PELC urbano, PELC Povos de Comunidades Tradicionais e PELC Vida Saudável, não havendo distinção entre as modalidades e entre os municípios participantes.

\section{Resultados E Discussão}

Das propostas avaliadas, 199 tiveram avaliações abaixo de 50 pontos. Isso significa que a falta de competência técnica à apresentação das propostas atinge $1 / 5$ das prefeituras que participaram dos Editais de 2013. Por outro lado, a não adesão aos Editais de 2013 foi de $82,2 \%$.

O semiárido brasileiro é a macrorregião com maior vulnerabilidade socioeconômica do país (MELO; PEREIRA; DANTAS NETO, 2009), ou seja, concentra um grande número de municípios ( $\mathrm{n}=1.126: 20,22 \%$ do total) que são carentes de políticas públicas, principalmente as sociais. Aproximadamente $23 \%$ dos municípios do semiárido brasileiro participaram dos editais 001 e 002 do PELC 2013, isto é, a taxa de não adesão atingiu 76,91\% para os municípios da região semiárida (Tabela 1). Isso significa que variáveis importantes se interpõem entre as prefeituras que são alvos prioritários de políticas sociais e a apresentação de propostas.

As prefeituras do semiárido, além de terem uma taxa de não adesão ao programa da ordem de $77 \%$, cerca de um quarto das propostas não possuem condições de competir pela implementação do programa. Dentre o total de prefeituras que não alcançaram 50 pontos, as do semiárido são responsáveis por 33,73\% do resultado.

O semiárido é um fator que impacta negativamente na apresentação de propostas. São prefeituras que carregam uma dupla desvantagem: participar do semiárido e não ter capacidade técnica para concorrer aos Editais 2013 do PELC.

O papel da capacidade técnica na restrição do acesso às políticas públicas já havia sido destacado por Melo (1996), ao mencionar que a excepcional heterogeneidade dos cerca de 5 mil municípios brasileiros, no plano socioeconômico e no que se refere à capacidade de gestão das prefeituras quanto a recursos humanos, infraestrutura material, tamanho e renda, constitui-se num grande impedimento para a implantação de políticas concebidas como se todos os municípios fossem iguais em suas capacidades.

Oliveira (2006, p. 280) aponta que

a capacidade técnica de gestionar o planejamento de políticas públicas é apontado como outro limitador dos países em desenvolvimento. Faltam recursos humanos capacitados e motivados, equipamentos, experiência e competência técnica dos órgãos responsáveis para planejar as políticas públicas.

Se levarmos em conta que o semiárido é a macrorregião com maior vulnerabilidade socioeconômica do país, podemos inferir que as condições apontadas por Oliveira (2006) são maximizadas nesse contexto e impedem que o Ministério do Esporte atue de forma a garantir o que está previsto no Inciso 3 do Art. 3 da CF/88.

5 IPTU, Imposto Predial e Territorial Urbano; ISS, Imposto Sobre Serviços; ITBI, Imposto sobre a Transmissão Inter-Vivos de Bens Imóveis.

6 A aplicação do FPM tem efeito redistributivo. Melhora o aporte financeiro dos municípios mais vulneráveis e menores. 
Durante a última década, o Brasil conseguiu melhorar a posição dos municípios em relação ao IDH. Mesmo assim, mais de $25 \%$ dos municípios ainda se encontram entre os níveis Muito Baixo $(0,0$ a 0,499) e Baixo de $\operatorname{IDH}(0,500$ a 0,599). Desses municípios carentes de políticas sociais $15,86 \%(\mathrm{n}=222)$ apresentaram propostas aos editais do PELC no ano de 2013.

A taxa de não adesão entre os municípios de menor IDH foi de $84,14 \%$. As prefeituras de municípios de menor IDH que apresentaram propostas e alcançaram pontuação abaixo de 50 foi de $26,12 \%(n=58)$. Isso significa que $1 / 4$ das propostas dos municípios de menor vulnerabilidade foi descartada por falta de capacidade técnica na apresentação das proposições. Para essas prefeituras, a falta de capacidade técnica as impede de ter acesso à política social.

Outra característica da vulnerabilidade dos municípios brasileiros está associada ao seu tamanho (RUBIRA, 2013; JESUS, 2011). Municípios menores, com até 20.000 habitantes, principalmente das regiões Norte e Nordeste, carregam múltiplas vulnerabilidades. No Brasil, esse grupo representa 70,27\% $(\mathrm{n}=3.914)$ do total de municípios.

Os municípios pequenos foram responsáveis por pouco mais de $16 \%$ das propostas desse grupo $(n=631)$. A taxa de não adesão aos Editais de 2013 do PELC foi de 83,87\%. São municípios que deveriam ser alvos de políticas sociais, mas que não respondem ativamente aos incentivos estabelecidos pelos editais. $\mathrm{O}$ número de propostas desses municípios que não alcançaram a nota de corte estabelecida aqui foi de $137(21,71 \%)$.

Não obstante, uma baixa adesão ao Edital do PELC revela que os municípios pequenos não possuem capacidade técnica para apresentar projetos que superem 50 pontos. São municípios que reúnem, novamente, a dupla desvantagem: ser um município do semiárido e não possuir capacidade técnica.

Mesmo o federalismo fiscal brasileiro sendo um dos mais descentralizados do mundo (SOUZA, 2004), uma parcela importante dos municípios não possui capacidade de arrecadação capaz de fazer frente aos desafios colocados pelas políticas sociais, produzindo, assim, novas distorções, como a ausência de burocracias com alta qualificação (MELO, 1996). São municípios dependentes das transferências intergovernamentais, principalmente o FPM. Com esse recurso, os governos municipais têm mais possibilidades de constituírem seus governos e suas políticas públicas.

O número de municípios brasileiros dependentes do FPM pelos critérios aqui sinalizados é de 3.693 (66,3\%). Desses, 623 apresentaram propostas para descentralização do PELC, alcançando taxa de não adesão aos Editais de 2013 do PELC de 83,12\% (Gráfico 4), taxa muito elevada para municípios que compõem o campo de prioridade do programa. Mais uma vez, destaca-se aqui a não adesão como uma das fragilidades do programa.

Dos municípios que apresentaram proposta, 134 não tiveram capacidade técnica para superar os 50 pontos na avaliação. $\mathrm{O}$ ator descentralizador privilegiado (por ser dependente de recursos federais) do programa não possui capacidade para apresentar um bom projeto.

Do ponto de vista regional, a incapacidade de apresentar projetos que superem os 50 pontos também reforça nossa preocupação com o desenho do programa. As regiões Norte e Nordeste possuem $40,27 \%$ do total de municípios brasileiros. Porém, essas regiões são responsáveis por $51,75 \%$ dos projetos com menos de 50 pontos. As regiões mais carentes estão superdimensionadas nesse aspecto, revelando terem problemas para participarem de uma concorrência em que todos estão em condições de igualdade.

Aqueles municípios que seriam alvos prioritários de políticas sociais acabam sendo preteridos - ao menos uma parcela deles - por conta de dificuldades na apresentação de propostas para participação dos Editais do PELC de 2013. A falta de capacidade técnica, critério pelo qual esses municípios são julgados, é um dos marcadores de sua vulnerabilidade, e o desenho do programa é incapaz de lidar com essa realidade. 
Ao investigar o campo da saúde, Bodstein (2002) aponta que os municípios mais pobres e inteiramente dependentes dos repasses federais apresentam capacidade técnica limitada para planejar, organizar e montar uma estrutura de serviços.

Os municípios situados nas regiões e estados mais desenvolvidos tendem a reunir os requisitos básicos necessários para assumirem a gestão descentralizada e o desenvolvimento de ações mais complexas, já que, teoricamente, dispõem de maior capacidade técnica e gerencial, bem como maior aporte de recursos financeiros (BODSTEIN, 2002). Dos municípios que conquistaram pontuação acima de 90 pontos $63,16 \%(\mathrm{n}=114)$ são das regiões Sul e Sudeste do Brasil.

Os mais vulneráveis têm mais dificuldades para apresentarem projetos em condições de competitividade com outras prefeituras. As variáveis aqui destacadas têm impacto diferenciado entre as propostas que alcançaram mais de 90 pontos: 57,89\% são de municípios com menos de 20 mil/hab., mas 2/3 delas são das regiões Sul e Sudeste; 57,02\% são de municípios dependentes do FPM, porém, 61,54\% são das regiões Sul e Sudeste; $16,67 \%$ são de municípios de baixo IDH e $91 \%$ deles são da região Nordeste; e 18,42\% são do semiárido.

\section{Considerações Finais}

A postura conservadora do estudo ao indicar a nota de corte de 50 pontos busca conhecer aqueles municípios com maiores dificuldades na elaboração do projeto, que poderia ter ampliado até a nota 70 e, mesmo assim, estaria considerando proponentes que não tiveram suas propostas implementadas.

A literatura reconhece que a falta de capacidade técnica é um dos marcos das desigualdades entre os municípios brasileiros. Diante da exiguidade de recursos para implementação da política, estabelecer critérios técnicos para seleção dos municípios promove desigualdade que a política pública (PELC) deveria combater.

O Mapa Esportivo do IBGE no ano de 2017 mostrou com bastante nitidez que há carência de políticas públicas de esporte e lazer nos municípios brasileiros, principalmente no interior do país. Portanto, toda a política que vem para superar essa realidade contribui para a diminuição das desigualdades. Sendo assim, chama a atenção, escolhendo qualquer marcador de vulnerabilidade, o elevado percentual de municípios que não fizeram propostas aos editais aqui analisados.

Diante dos resultados apresentados, é pouco produtivo colocar a responsabilidade nos competidores aos Editais do PELC no ano de 2013. As prefeituras, como é de se esperar numa federação altamente desigual como a brasileira, não apresentam condições de participarem do edital com equidade. A falta de capacidade técnica é resultado da desigualdade estrutural da sociedade brasileira e não pode ser transformada em elemento qualificador dos proponentes.

O modelo de descentralização do PELC, aqui em análise, em vez de promover municípios vulneráveis, operou no sentido de criar dificuldades adicionais a esses municípios que deveriam ser implementadores prioritários do programa. O desenho do programa não colabora para que a União cumpra o que está previsto no Inciso 3 do Art. 3 da CF/88.

Quando na média da não adesão, a maioria dos municípios com alguma vulnerabilidade não apresenta propostas em razão de: (a) o programa não atende as necessidades dos proponentes; (b) os incentivos são insuficientes para mobilizar a atenção dos proponentes; (c) as informações não chegam de forma qualificada entre os potenciais implementadores. Sendo vulneráveis e carentes de políticas sociais, todas essas variáveis deveriam ser equacionadas pelo programa. O cumprimento do Inciso 3 do Art. 3 da CF/88 exige tratamento desigual de forma a promover maior igualdade de acesso, como já destacaram análises de programas federais do tipo top-down. 
No federalismo brasileiro não há instrumentos normativos ou políticos capazes de pressionar entes subnacionais (municípios) a aceitarem a agenda do governo federal. Portanto, apresentar propostas para descentralizar um programa de um ente superior (descentralização top-down) envolve decisão autônoma dos custos de oportunidade. Apresentaram propostas aos editais do PELC/2013 992 municípios, cerca de 17\% do total de municípios brasileiros (n $=5.570$ ).

A grande maioria das cidades brasileiras prefere a não adesão. Considerando que os municípios podem realizar gastos na Função Desporto e Lazer (FDL) com recursos próprios, a não adesão, em tese, não significa inação. No entanto, compreender as motivações que levam a esse comportamento pode aprimorar o desenho do programa.

\section{Referências}

ALMEIDA, M. H. T. Federalismo e políticas sociais. Revista Brasileira de Ciências Sociais, São Paulo, v. 10, n. 28, p. 88-108, 1995.

AREIAS, K. T. V.; BORGES, C. N. F. As políticas públicas de lazer na mediação entre estado e sociedade: possibilidades e limitações. Revista Brasileira de Ciências do Esporte, v. 33, p. 573-588, 2011.

ARRETCHE, Marta. Federalismo e igualdade territorial: uma contradição em termos? DadosRevista de Ciências Sociais, Rio de Janeiro, v. 53, n. 3, 2010.

ARRETCHE, Marta. Federalismo e políticas sociais no Brasil: problemas de coordenação e autonomia. São Paulo em Perspectiva, São Paulo, v. 18, n. 2, p. 17-26, 2004.

ARRETCHE, Marta. Políticas sociais no Brasil: descentralização em um Estado federativo. Revista Brasileira de Ciências Sociais, São Paulo, v. 14, n. 40, p.111-141. jun. 1999.

ARRETCHE, Marta. Políticas sociais no Brasil: descentralização em um Estado federativo. Revista Brasileira de Ciências Sociais, v. 14, n. 40, p. 111-141, 1999.

ARRETCHE, M.; VAZQUEZ, D.; GOMES, S. Descentralização e autonomia: deslocando os temos do debate. In: LAVALLE, A. G. (Org.) Horizonte da política: questões emergentes e agendas de pesquisa. São Paulo: Editora Unesp: Cebrap: CEM, 2012. p.29-96.

BODSTEIN, R. Atenção básica na agenda da saúde. Ciência \& Saúde Coletiva, v. 7, n. 3, p. 401-12, 2002.

IBGE. Instituto Brasileiro de Geografia Estatística. Perfil dos municípios brasileiros: esporte, 2017.

CASTRO, S. B. E. Políticas públicas para o esporte e lazer e o ciclo orçamentário brasileiro (2004-2011): prioridades e distribuição de recursos durante os processos de elaboração e execução orçamentária. 2016. 382 f. Tese (Doutorado em Educação Física) Universidade Federal do Paraná, Programa de Pós-Graduação em Educação Física, Curitiba, 2016.

FLEURY, Sônia. Políticas sociais e democratização do poder local. In: VERGARA, Sylvia Constant; CORREA, Vera L. A. (Org.). Propostas para uma gestão pública municipal efetiva. 2.ed. Rio de Janeiro: Ed. FGV, 2004. p. 91-115. 
GOMES, G. M; DOWELL, M. C. Descentralização política, federalismo fiscal e criação de municípios: o que é mau para o econômico nem sempre é bom para o social. Texto para Discussão n. 706, IPEA, Brasília, 2000.

JESUS, A. C. Desafios e Diversidades na Gestão Programa Bolsa Família em Pequenos e Médios Municípios. In: JORNADA INTERNACIONAL DE POLÍTICAS PÚBLICAS, 2011, SÃO LUÍS, MA. V JORNADA INTERNACIONAL DE POLÍTICAS PÚBLICAS ESTADO, DESENVOLVIMENTO E CRISE DO CAPITAL, 5., 2011, Maranhão. Anais... São Luis, Ma.: Universidade Federal do Maranhão, 2011.

KERSTENETZKY, C. L. Por que se importar com a desigualdade. Dados-Revista de Ciências Sociais, Rio de Janeiro, v. 45, n. 4, p. 649-675, 2002.

MEDEIROS, M. DINIZ, D. Paradigmas de justiça distributiva em políticas sociais. Revista de Estudos Universitários, Sorocaba, SP, v. 34, n.1. p. 19-31, 2008.

MELO, J. A. B. de; PEREIRA, R. A.; DANTAS NETO, J. Atuação do Estado brasileiro no combate à seca no Nordeste e ampliação das vulnerabilidades locais. Qualitas Revista Eletrônica, [S.1.], v. 8, n. 2, set. 2009. http://dx.doi.org/10.18391/qualitas.v8i2.387.

MELO, M. A. O sucesso inesperado das Reformas de Segunda Geração: federalismo, reformas constitucionais e política social. Dados-Revista de Ciências Sociais, Rio de Janeiro, v. 48, n. 4, p. 845-889, 2005.

MELO, M. A. Crise federativa, guerra fiscal e "hobbestanismo municipal": efeitos perverso da descentralização? São Paulo em Perspectiva, São Paulo, v. 10, n. 3, p. 11-20, 1996.

OLIVEIRA, J. A. P. Desafios do planejamento em políticas públicas: diferentes visões e práticas. Revista de Administração Pública, Rio de Janeiro, v. 40, n. 1, p. 273-288, mar. labr. 2006.

PAES, N. L.; SIQUEIRA, M. L. Desenvolvimento regional e federalismo fiscal no Brasil: em busca da igualdade na distribuição de receitas. Economia Aplicada, v. 12, n. 4, p. 707-742, out. /dez. 2008.

RUBIRA, I. M. M. Administração e gestão de serviços sociais públicos: um olhar sobre os pequenos municípios. Revista Ampla de Gestão Empresarial, São Paulo, v. 2, n. 2, p 97$108,2013$.

SABATIER, Paul A. Top-down and bottom-up approaches to implementation research: a critical analysis and suggested synthesis. Journal of Public Policy, v. 6, n. 1, p. 21-48, 1986.

SANTOS, E. S. Análise do Desempenho do Edital de 2012 do PELC. Licere, Belo Horizonte, v. 20, n. 3, p. 41-58, 2017.

SANTOS, Edmilson Santos; STAREPRAVO, Fernando Augusto; SOUZA NETO, Marina Silva Souza. Programa Segundo Tempo e o vazio assistencial na região Nordeste. Movimento, v. 21, n. 3, p. 759, 2015.

SOUSA, E. S. Sistema de monitoramento \& avaliação dos programas Esporte e Lazer da Cidade e Segundo Tempo do Ministério do Esporte. Belo Horizonte: O Lutador, 2010. 
SOUZA, C. Federalismo e Descentralização na Constituição de 1988: Processo Decisório, Conflitos e Alianças. Dados-Revista de Ciências Sociais, Rio de Janeiro, v. 44, n. 3, p. 513 560,2001

SOUZA, Celina. Governos locais e gestão de políticas sociais universais. São Paulo em perspectiva, v. 18, n. 2 , p. 27-41, 2004.

STAREPRAVO, F. A.; MARCHI JÚNIOR, W. Aspectos técnicos, conceituais e políticos do surgimento e desenvolvimento do Programa Esporte e Lazer da cidade. Pensar a Prática, Goiânia, v. 16, p. 923- 941, 2013.

TORRES, H. G. et al. Pobreza e espaço: padrões de segregação em São Paulo. Estudos Avançados, São Paulo, v. 17, n. 47, p. 97-128, 2003.

TREVISAN, A. P.; BELLEN, H. M. Avaliação de políticas públicas: uma revisão teórica de um campo em construção. Revista de Administração Pública, Rio de Janeiro, v. 42, n. 3, p. 529-550, maio/jun. 2008.

VIEIRA, Adriana Antunes et al. Programa Esporte e Lazer da Cidade-PELC: Interfaces Entre o PELC e as Desigualdades Sociais. Licere, Belo Horizonte, v. 20, n. 1, p. 161-180, 2017.

VIEIRA, L. H. S. Estudos sobre a gestão do programa esporte e lazer da cidade. 2010. 184 f. Dissertação (Mestrado em Administração) - Universidade Federal de Viçosa, Viçosa, 2010.

Recebido em: 09/05/2018

Revisado em: 08/08/2018

Aprovado em: 24/09/2018

Endereço para correspondência:

edmilson.santos@univasf.edu.br

Edmilson Santos dos Santos

Universidade Federal do Vale do São Francisco, Colegiado de Educação Física.

Av. José de Sá Maniçoba, S/N

Centro

56304917 - Petrolina, PE - Brasil 